\title{
Research Trend of Technology Management
}

Yoko Ishino

GraduateSchool of Innovation \& Technology Management, Yamaguchi University

Address: 1677-1 Yoshida, Yamaguchi, 753-8511, Japan

E-mail: ishino.y@yamaguchi-u.ac.jp

\begin{abstract}
Many business organizations are eager to continually create innovation and contribute to the society. For this purpose, companies should not only pursue the leading-edge technology but also understand the nature of technology and manage their technological fundamentals to create competitive advantage. Management of Technology (MOT) is a typical research field for this issue. MOT covers many contents including administrative strategy, R\&D management, manufacturing management, production control, marketing, accounting, finance, intellectual property strategy, business ethics, and others. By applying a text-mining method to the conference proceedings of IAMOT (International Association for Management of Technology), this study examines the research trends in MOT, detects a change in them that have occurred over time, and finally considers the effects of the social situation on academic research.
\end{abstract}

Keywords: Text Mining, Network Analysis, Technology Management, MOT

Paper received: 19/11/2013

Paper accepted for Publication: 31/01/2014 


\section{INTRODUCTION}

IManagement of Technology (MOT) is defined as the disciplines of management that enable organizations to manage their technological fundamentals to create competitive advantage. MOT should not only fulfill the management needs of a specific set of technologies within a domain and inter-domain relationship, but it should also develop the implementation strategies according to the available resources, current technologies, future markets, and socio-economic environment [1]. Therefore, how to manage technology has become an important issue in the past few decades, and the MOT community has developed a wide range of methodologies and applications for both academic research and practical applications [2,3]. Nowadays, MOT covers a wide range of contents including administrative strategy, R\&D management, manufacturing management, production control, marketing, accounting, finance, intellectual property strategy, business ethics, and others. MOT research has been conducted at various levels for each kind of these contents. This made it difficult to understand an overall picture of the MOT research field. Finding research trends, or historically critical topics, in MOT is useful for understanding the key concepts of current MOT.

In this paper, we studied the research trends in MOT by applying a text-mining method to the conference proceedings of IAMOT (International Association for Management of Technology). We detected a change in the research stream and finally found the effects of the social situation on the research trends.

\section{RESEARCH OBJECTIVE}

There seems to be several methods for investigating research trends in technology management. You might conceive of a method which surveys the trends in papers published in some academic journals. However, since each academic journal has its own predetermined themes, it is difficult to grasp the overall trend of MOT by just reviewing a few journals. Moreover, since research presented in academic journals is already finished, there is a time lag between research run currently and research presented in journals.

Another method to investigate the research trends is to examine the topics presented in major international conferences on MOT. Such conferences are likely to reflect research trends without the time lag found in academic journals, and their proceedings would appear to be an appropriate research target. The problem is how to investigate them. In an international conference, not all presentations are assigned to appropriate sessions, since they are constrained by time and location. In other words, the number of presentations included in a session category does not give an accurate picture of research trends. In addition, the session categories themselves change every year, reflecting the opinions of the program committee.

In light of this situation, this study focuses on the content discussed at an international MOT conference, deliberately ignoring the assigned session categories. We have analyzed the abstract texts in the proceedings of the International Association for Management of Technology (IAMOT) conferences, in order to find how research trends have changed over time. IAMOT is a non-profit, non-governmental professional association in the USA whose purpose is to encourage high quality research and education in the field of MOT. IAMOT's first international conference was held in 1988, and its 21st conference, which was held in Taiwan in 2012, had approximately 400 participants from 34 countries around the world. The IAMOT conference is one of the 
mostpredominant international academic conferences concerning MOT.

The session categories in IAMOT 2003 were as follows.

- $\quad$ The session categories in IAMOT 2003 were as follows.

- $\quad$ Knowledge Management

- $\quad$ Strategic Competencies for Sustainable Development

- Social Impact of Technologies Development

- MOT Education and Research/ Corporate Universities

- Innovation and New Product Development

- $\quad$ National Systems for Technology Development

- Small Businesses and Entrepreneurship/ Technology Incubation

- $\quad$ Emerging and Breakthrough Technologies

- Technology Transfer/ Technology and Security

- $\quad$ Technology Foresight and Forecasting

- Information and Communication Technology Management

- The Integration of Technology and Business Strategies

- R\&D Management

- $\quad$ Project Management

- Industrial and Manufacturing Systems Technologies/ Supply Chain Management

- Virtual Organizations and Partnerships/ E-Commerce

- $\quad$ MOT in Developing Countries

- Managing R\&D in China

- $\quad$ By contrast, the list of the session category in IAMOT 2012 was indicated below.

- Technology-Service Convergence

- $\quad$ MOT in Services

- $\quad$ R\&D Management

- Technology Strategy

- Technology Transfer

- Service Innovation

- Logistics and SCM

- Managing Energy Technology

- ICT Management

- $\quad$ Science and Technology Policies

- $\quad$ Science and Technology Incubation and Entrepreneurship

- $\quad$ Science, Technology and Society

- Management of Technology in Developing Country

- MOT Education and Research

- Manufacturing Servitization

- Measurement of Technology

- User Innovation and Open Innovation in East Asia

There is a large difference between the two session category lists.

This study aims to reveal the changes in the MOT research trends by performing textmining approach on the IAMOT conference proceedings, and to consider the impact of societal changes on academic research. 


\section{ANALYSIS METHODS}

\section{Data Used for Analysis}

For the data to be used in our experiment, we extracted the abstracts from the research papers included in the proceedings of the IAMOT conferences held in 2003, 2008, and 2012. Almost all papers had explicitly the abstract. However, some papers were free from boundaries between the abstract and the body text. In such cases, we determined by hand the text part corresponding to the abstract.During this process, we excluded any papers for which an abstract was clearly omitted.

\section{Methods}

First, we performed morphological analysis on the abstract texts using one of part-ofspeech taggers [4]. Then, focusing on only the nouns (general nouns and proper nouns), we calculated numerical feature values of each noun including the frequency of appearance of a word and the co-occurrence of words. Finally, we investigated the relationships between words using network analysis.

\section{RESULTS}

\section{Appearance Frequency of Words}

The numbers of abstracts for each conference year were as follows: 369 in 2003, 236 in 2008, and 207 in 2012-making a total of 812 abstracts in all. There was some variation in the lengths of the abstracts, with the average being 246 words.

Table 1 shows the top 50 ranking words that had high appearance frequency within the 812 files (nouns and proper nouns). We ignored the words "paper," "study," "research," and "result" since these are common to all academic paper abstracts, regardless of the research field. Table 1 shows that the following words that express characteristics of MOT had the highest ranking: "technology," "process," "development," "innovation," "management," "product," and "market." This result is adequate but insipid, since these words are clearly and directly related to MOT.

Then, we investigated the frequency distribution of the appearance of words.The results show that several dozen words from the top in the appearance frequency recorded very high frequency, while the overwhelming majority of words appeared only a few times. 
Table 1 Top $\mathbf{5 0}$ Words for Appearance Frequency in Total Abstracts

\begin{tabular}{|c|c|c|c|c|c|}
\hline Ranking & Word (Nown) & $\begin{array}{c}\text { Appearance } \\
\text { Ratio }\end{array}$ & Ranking & Word (Nown) & $\begin{array}{c}\text { Appearance } \\
\text { Ratio }\end{array}$ \\
\hline 1 & technology & 51.7 & 26 & datum & 19.7 \\
\hline 2 & process & 42.9 & 27 & service & 19.3 \\
\hline 3 & development & 41.7 & 28 & literature & 19.2 \\
\hline 4 & innovation & 37.6 & 29 & performance & 19.0 \\
\hline 5 & management & 34.1 & 30 & country & 18.8 \\
\hline 6 & company & 34.0 & 31 & way & 18.3 \\
\hline 7 & product & 31.9 & 32 & role & 18.0 \\
\hline 8 & model & 31.0 & 33 & res curce & 17.6 \\
\hline 9 & industry & 29.7 & 34 & environment & 17.4 \\
\hline 10 & matket & 29.1 & 35 & method & 17.4 \\
\hline 11 & firm & 28.2 & 36 & time & 17.1 \\
\hline 12 & business & 27.8 & 37 & need & 16.7 \\
\hline 13 & analysis & 27.8 & 38 & vahe & 16.5 \\
\hline 14 & case & 27.2 & 39 & $R \& D$ & 16.5 \\
\hline 15 & system & 27.0 & 40 & issue & 16.1 \\
\hline 16 & strategy & 26.5 & 41 & change & 16.0 \\
\hline 17 & lnow ledge & 24.8 & 42 & concept & 15.8 \\
\hline 18 & factor & 24.0 & 43 & problem & 15.8 \\
\hline 19 & information & 23.2 & 44 & sector & 15.8 \\
\hline 20 & order & 23.0 & 45 & impact & 15.4 \\
\hline 21 & approach & 22.0 & 46 & use & 15.1 \\
\hline 22 & project & 22.0 & 47 & framew ork & 15.1 \\
\hline 23 & activity & 21.4 & 48 & capabjlity & 15.0 \\
\hline 24 & level & 21.4 & 49 & policy & 14.8 \\
\hline 25 & organization & 21.2 & 50 & effect & 14.5 \\
\hline
\end{tabular}

\section{Co-Occurrence of Words}

Words can be regarded as compositional units for expressing what an author would like to state. Since the words existing in the same abstract shape together the abstract according to author's aim, such relationships between words are very important. If any two nouns appear in the same abstract, we defined the relationship of them as the co-occurrence. The strength of the co-occurrence can be represented by the number of the abstract in which the co-occurrence is observed. By using a graphical network, we expressed the state of the co-occurrence of words in each conference year. In the network, each word is depicted as a node, and co-occurrent relationships are represented by edges. The edges are drawn when the strength of the cooccurrence exceeds a certain threshold value. We calculated the various feature values of each word network in each conference year, such as the density, degree centrality, closeness centrality, betweenness centrality, and so on. We found that the feature values are very similar between the word networks of each conference year, since the networks share a similar structure with each other.Moreover, the average distance is short, and the cluster coefficient is high, showing that the networks are small-world networks. Next, we found that the frequency distribution of node degrees (the number of nodes to which a given node is connected) is exponential, and that the networks are scale-free networks. This was observed in every conference year. 


\section{Observed Research Trends}

In investigating changes in research trends, we focused on the betweenness centrality of the network nodes. The betweenness centrality of a node indicates the ratio of the edges between all other pairs of nodes in which that node is included. This metric is proposed based upon the notion that the more routes that pass through a point, the higher its betweenness centrality will be. In this research, the betweenness centrality of a word becomes higher when the word is co-occurrent with more other kinds of words. Table 2 shows the top 20 words for each conference year in terms of the betweenness centrality. What is interesting to note is that in 2012, the word "patent" appeared for the first time, in 8th place. We discuss this point below in greater detail.

First, as shown in Figure 1, we created the co-occurrence network of words in the case of IAMOT 2012, representing the betweenness centrality by the size of the nodes. The large node at the bottom right represents "technology," and the large node at the top right is "innovation." These two nodes have extremely high betweenness centrality. However, "patent" (a mediumsized node in the bottom left corner) also has a moderate degree of influence. Next, we investigated how the appearance rate of the word "patent" had changed from one conference year to another. The results showed the following increasing trend: $2.2 \%$ in $2003,6.8 \%$ in 2008 , and $14.0 \%$ in 2012.

There were 29 papers that included the word "patent" in their abstracts in 2012. The majority of these did not include "patent" in the title, and they were spread across the session categories. Moreover, the existing session categories did not have any categories that specialized patent analysis.

The reason why the MOT research that relates to patents increased can be that the progress of the ICT technology permitted enhancement of the data processing ability and development of the patent analysis tools. This might indicate a new and growing trend where people come to use more of the patent analyses in making business strategy.

Table2 Top 20 Words for Betweenness Centrality

\begin{tabular}{|c||ll||ll|l|l|}
\hline Ranking & \multicolumn{2}{|c||}{ in 2003 Conference } & \multicolumn{2}{c|}{ in 2008 Conference } & \multicolumn{2}{c|}{ in 2012 Conference } \\
\hline 1 & technology & 0.252 & technology & 0.216 & technology & 0.303 \\
\hline 2 & process & 0.206 & process & 0.120 & innovation & 0.230 \\
\hline 3 & development & 0.110 & development & 0.109 & model & 0.089 \\
\hline 4 & management & 0.060 & mana gement & 0.092 & development & 0.061 \\
\hline 5 & company & 0.054 & innovation & 0.070 & process & 0.054 \\
\hline 6 & product & 0.040 & company & 0.043 & analysis & 0.044 \\
\hline 7 & market & 0.028 & industry & 0.036 & firm & 0.030 \\
\hline 8 & project & 0.025 & product & 0.036 & patent & 0.026 \\
\hline 9 & system & 0.021 & know ledge & 0.030 & performance & 0.023 \\
\hline 10 & innovation & 0.014 & business & 0.020 & management & 0.018 \\
\hline 11 & model & 0.014 & factor & 0.018 & policy & 0.016 \\
\hline 12 & organization & 0.013 & analysis & 0.015 & indus try & 0.016 \\
\hline 13 & business & 0.012 & approach & 0.014 & strategy & 0.014 \\
\hline 14 & information & 0.012 & case & 0.013 & company & 0.013 \\
\hline 15 & strategy & 0.009 & market & 0.012 & datum & 0.013 \\
\hline 16 & chain & 0.008 & ife & 0.011 & literature & 0.012 \\
\hline 17 & order & 0.008 & strategy & 0.011 & case & 0.010 \\
\hline 18 & firm & 0.008 & leve1 & 0.010 & business & 0.008 \\
\hline 19 & industry & 0.007 & project & 0.009 & lnowledge & 0.006 \\
\hline 20 & sector & 0.006 & firm & 0.009 & prodvct & 0.005 \\
\hline
\end{tabular}




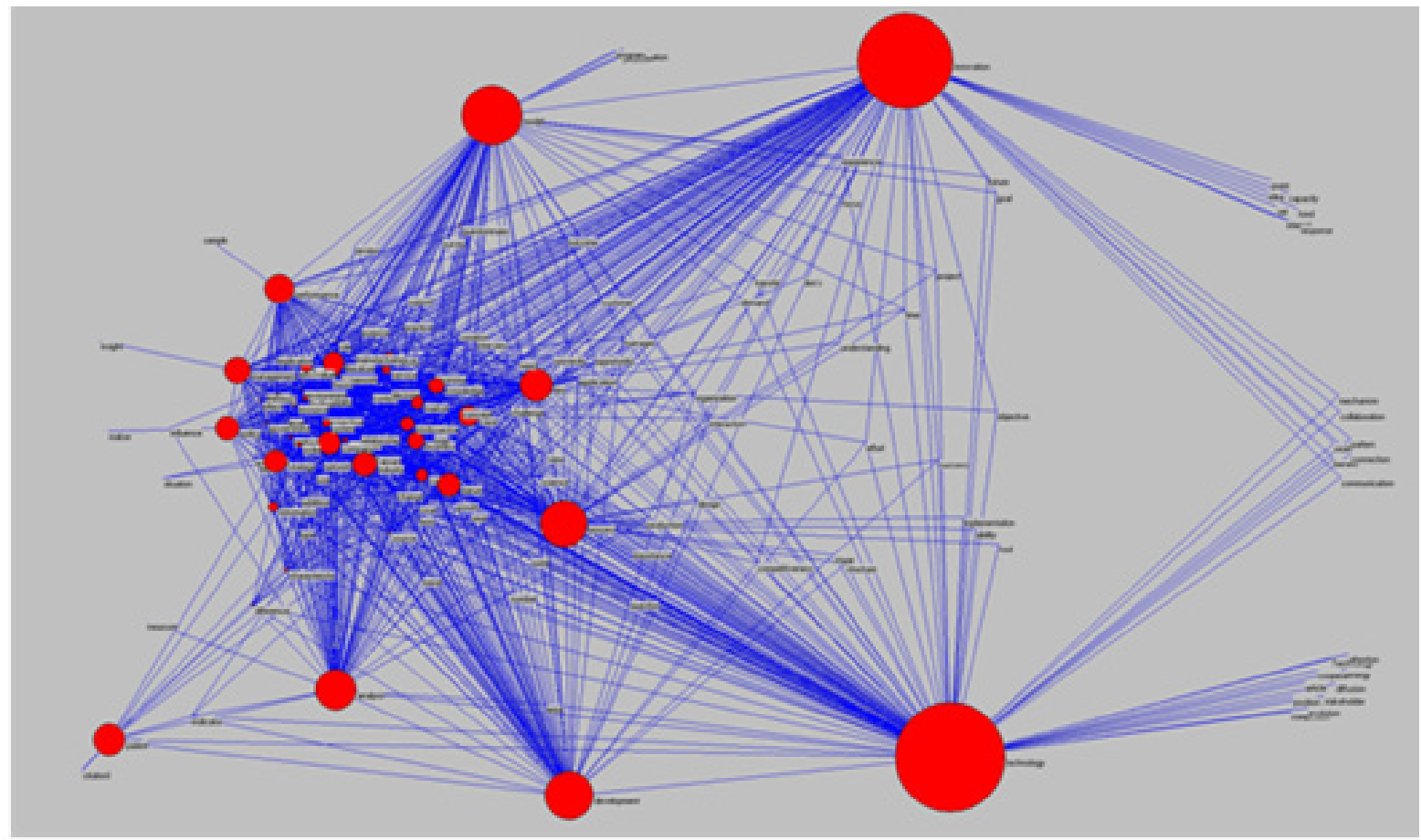

Figure 1 Co-occurrence Network of Words in 2012 Conference

We have shown, then, that patent-related research has been increasingly presented at the IAMOT conference. We can further say that it would not have been possible to discover this phenomenon from the conference session titles alone. This demonstrates the effectiveness of the proposed method, which combines the text mining approach with the network analysis of the co-occurrence of words.

\section{RELATED RESEARCH}

\section{Topic Extraction Using Text Mining}

Research and development in the field of text mining has increased and spread since the rapid digitalization of text began in the late 1990s [5]. One field of application for text mining involves topic extraction [6]. This is the automatic extraction of the appropriate main topics from a group of texts after natural language processing, in order to assist with text sorting. Currently, mechanical topic extraction methods are generally based on a vector space model, in which significant clusters of words that appear in a text are conceived as the base of a feature space, and each text document is treated as a word frequency vector. This study employs concepts that are similar to topic extraction, in which a text is characterized using a vector that represents the appearance or non-appearance of words. However, this study does not select beforehand a set of words that characterizes the topic.A typical research of the topic extraction uses a training dataset where documents for training are already classified into topics before learning [7].That is called the supervised learning, whereas this study, by contrast, does not aim to learn the classification of a training set. 


\section{Co-Occurrence Network of Words}

In the framework of a co-occurrence network, words that appear in a document are treated as nodes, and words that appear in proximity to each other are linked by edges.In such a network, words that have a significant degree of relatedness form solid mutual connections called cluster structures. On the other hand, the connections between words with a low degree of relatedness are sparse. Several methods have been proposed for grasping the meanings of words and for eliminating polysemy, by focusing on this characteristic of density of connections in network structure [8]. Recently, a complex network approach has been used to express the relationships between factors, in an attempt to investigate not only the static characteristics, but also the dynamics of factors. Previous research demonstrated how well the core words of a language (the kernel lexicon) could be extracted based on the difference in the exponential distribution of the co-occurrence network of words[9].Another research investigated the difference in the formation of the cluster structure appeared in the co-occurrence network of words in newspaper articles [10].

\section{CONCLUSION}

In this study, we studied the research trends in MOT by applying a text-mining method to the conference proceedings of IAMOT. By performing the network analysis of the co-occurrence of words, we detected a change in the research stream and finally found the influence of the social situation on the research trends. We have shown that patent-related research has been increased in MOT research. This phenomenon could not be discovered only from the conference session titles.

In the future, we will study the word co-occurrence networks in detail by using new indices representing word features, so that we may find the signs of the research trends.The insights obtained will then be able to be used to create effective educational materials of MOT.

\section{REFERENCES}

[1] Linn, R.J., Zhang, W., Li, Z.Y., An intelligent management system for technology management, Computers and Industrial Engineering, 2000, 38:397-412.

[2] Drejer, A., The discipline of management of technology, based on considerations related to technology, Technovation, 1997, 17(5): 253-265.

[3] Liao, S., Technology management methodologies and applications: A literature review from 1995 to 2003, Technovation, 2005, 25(4): 381-393.

[4] Schmid, H., Probabilistic part-of-speech tagging using decision trees. Proc of International Conference on New Methods in Language Processing, Manchester, UK, 1994.

[5] Nasukawa, T., Text mining: essence and usage of text mining on the basis of the technology and applied cases, Tokyo Denki University Press, 2006 (In Japanese) 
[6] Fukui, K., Saito, K., Kimura, M., Numao, M., Evaluation of Vector Representable Topics That were Extracted Automatically, Transactions on Mathematical Modeling and its Applications (TOM) in IPSJ (the Information Processing Society of Japan), 2007, 48(SIG6(TOM17)): 1-11. (In Japanese)

[7] Wayne, C., Multilingual topic detection and tracking: successful research enabled by corpora and evaluation, Proc. Language Resources and Evaluation Conference, 2000, 14871494.

[8] Ferret, O., Discovering word senses from a network of lexical cooccurrences, Proc. 20th international conference on Computational Linguistics, 2004, 1326-1332.

[9] Dorogovtsev, S.N. and Mendes, J.F.F., Language as an evolving word web, Proc. Royal Society of London, Series B, 2001, 268(1485): 2603-2606.

[10] Sato, S., Fukuda, K., Sugawara, T., Kurihara, S., On the Relationship between Word Bursts in Document Streams and Clusters in Lexical Co-occurrence Networks, Transactions on Database (TOD) in IPSJ (the Information Processing Society of Japan), 2007, 48(SIG14 (TOD35)): 69-81. (In Japanese) 